\title{
Spatially modulated phases in monolayers of mixed surfactants
}

\author{
Zhen-Gang Wang \\ Division of Chemistry and Chemical Engineering, California Institute of Technology, Pasadena, \\ California 91125
}

(Received 7 April 1993; accepted 25 May 1993)

\begin{abstract}
We present a theoretical study of spatially modulated phases in self-assembled monolayers of mixed surfactants. We propose two models which are appropriate, respectively, for monolayers at a fluid-fluid interface and monolayers on a solid substrate. We show that in both cases, the molecular shape asymmetry, coupled with the local composition variation, can lead to spontaneous formation of periodic structures. In the case of liquid-supported monolayers, the molecular shape asymmetry is manifested as a spontaneous curvature of each component of the film, which induces pcriodic variations both in the composition of the amphiphiles and in the height profile of the interface (ripples). In the case of solid-supported monolayers, the shape asymmetry is reflected in the spontaneous splay of the orientation of the amphiphiles, and the spatial modulation involves the composition as well as the orientation of the amphiphilic molecules. We analyze these models in some detail near the critical region, where we highlight the roles played by various length scales in determining the critical wavelength. We show that gravity has some very subtle and nontrivial effects for a liquid-supported, tension-free monolayer. We also present some preliminary results for the low temperature cases.
\end{abstract}

\section{INTRODUCTION}

Molecular monolayers formed by adsorption of amphiphiles at fluid-fluid or fluid-solid interfaces are of great practical interest because of their relevance to biological systems (e.g., cell membranes) and because of their technological potential as molecular electronic and optical devices. ${ }^{1}$ This practical interest, coupled with the fundamental interest for understanding quasi-two-dimensional systems, has sustained the research activity in the field for many decades. ${ }^{2} \mathrm{~A}$ unique feature of the monolayer systems is that the amphiphiles exist in three-dimensional space and yet the head groups (for most practical purposes) can be regarded as confined to a two-dimensional interface. This duality, and the intricate coupling between the conformational degrees of freedom (e.g., orientation of the amphiphile) and the translational degrees of freedom (e.g., positional arrangement of the head group), give rise to the very rich and complex structures and phase behaviors in these monolayer systems.

Closely related to the aforementioned duality is the special symmetry, or rather the lack thereof, in monolayers imposed by the inequivalence between the two sides of the interface (fiuid 1-fluid 2, fluid-solid) and between the head and tail parts of the amphiphiles. Some of the consequences of this special symmetry have been addressed by scveral authors. ${ }^{3-8}$ In particular, Chen et $a .^{4}$ using symmetry arguments, have shown that the usual isotropicnematic phase transition in a suspension of rods disappears when the rods are end anchored to an impenetrable surface.

Another effect due to the head-tail asymmetry is the so-called spontaneous curvature of the monolayer-a constraint-free, self-assembled monolayer usually forms a curved, rather than flat, geometry with some preferred radius of curvature (whose sign indicates whether the head or tail side of the monolayer is on the convex side).$^{9-11}$ The physical reason for the spontaneous curvature is the size and/or interaction length disparity between the head and tail groups of the amphiphilic chain, giving rise to some effective shape of the molecule. In fact, it is this effective shape of the molecule that determines to a large extent the shapes of the micellar aggregates of the surfactants (spheres, rods, bilayers, etc.), ${ }^{12}$ and the shapes of the emulsion droplets in microemulsions. ${ }^{13,14}$ When a monolayer is formed from adsorption of amphiphiles on the airwater interface, the overall flatness of the interface imposed by gravity, forces the monolayer to remain flat at the sacrifice of the spontaneous curvature. As a result, a dense monolayer at the air-water interface is usually frustrated because of this effect. This frustration can be relieved by adding another surfactant component with the opposite curvature. By appropriate spatial arrangement of the two species, cach component can achieve its spontaneous curvature to the extent allowed by the capillary force and the free energy cost due to composition inhomogeneity [see Fig. 1(a)].

When the amphiphiles reside at a fluid-solid interface, the head-tail asymmetry manifests itself in a different manner. Because the deformation free energy is usually rather high for a solid surface, the amphiphiles are constrained to have their head groups anchored on a flat surface. The only remaining degree of freedom is the orientation of the amphiphiles. (In a dense monolayer, the amphiphilic chains assume predominantly the trans conformation; hence to a good approximation, the flexibility of the chains can be neglected. ${ }^{15}$ ) In this case, the head-tail asymmetry gives rise to a spontaneous splay in the director of the molecules. Once again, however, for a single component monolayer, it is impossible to satisfy the spontaneous splay everywhere without having regions where the splay is of the wrong sign, and the free energy cost for having the latter usually cannot be compensated by the spontaneous splay free en- 


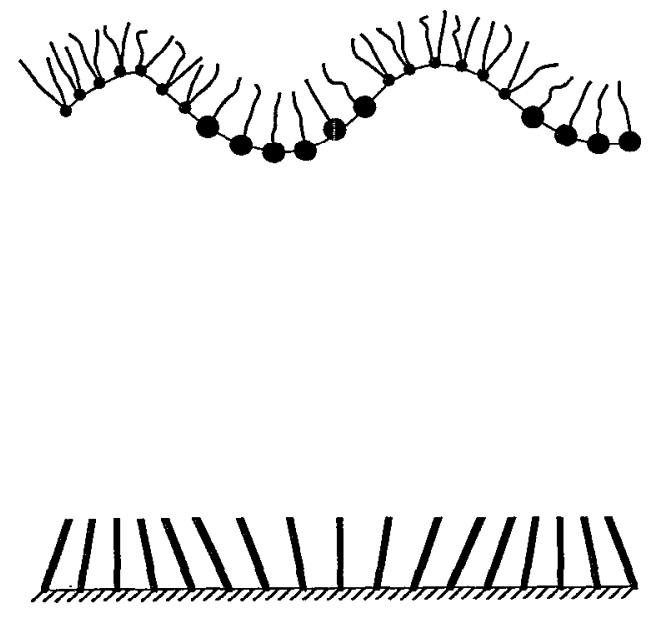

FIG. 1. A schematic cross-sectional view of (a) spontaneous rippling of a liquid-supported binary surfactant monolayer and (b) periodic variation of the molecular orientation in a solid-supported monolayer. The compositional variation in the latter case is not shown in (b).

ergy gain. Here then, a single component monolayer with head-tail asymmetry is again frustrated for having to maintain a zero-splay state. This frustration is relieved by adding another surfactant with the opposite spontaneous splay, which results in periodic variations in the orientation of the molecules [see Fig. 1(b)].

In this paper, we study the phenomenology associated with the structures and phase transitions in the two cases of mixed surfactant films, focusing in particular on the effects of spontaneous curvature or the spontaneous splay. This study is motivated by several considerations. First, in most applications, a surfactant species is often used along with a cosurfactant. Therefore, it is only natural to study the structures and phase behaviors of two or multicomponent surfactant monolayers. Second, the model systems studied here offer a good example to a general phenomenon in condensed-matter physics, namely, formation of spatial patterns induced by molecular asymmetry. A good understanding of this phenomenon may lead to the possibility of micromanipulating the arrangements of amphiphiles in some applications, such as in microelectronic or optical devices. Third, in the majority of phase transition phenomena in condensed matter, gravity usually has negligible effects. However, for the monolayer at the fluid-fluid interface, it plays an essential and subtle role. Finally, the systems studied here offer an interesting contrast between monolayers and bilayer vesicles ${ }^{16,17}$ of binary amphiphile mixtures. In the latter case, the two components reside preferentially on the two respective monolayers of the vesicles; thus one has a situation of phase separation without a phase boundary. ${ }^{18}$

Both monolayers at a fluid-fluid interface and at a fluid-solid interface will be studied in this paper. From a physical point of view, the two systems are of equal importance; hence a natural organization of this paper appears to be one in which the two systems are treated in parallel and equal amount of discussion is devoted to each subject. However, the approaches and discussions concerning these two systems are rather similar. Therefore, in the interest of avoiding undue repetition and saving space, we choose to discuss the fluid-fluid case in some detail and only briefiy treat the fluid-solid case. This consideration leads to an organization of the paper as follows: in Sec. II-IV, we give a detailed exposition to our study of monolayers at the fluid-fluid interface (henceforth referred to as liquidsupported monolayers). In Sec. II, we construct a Ginzburg-Landau free energy functional, incorporating the three major pieces of free energy contribution due to composition variation, curvature elasticity of the film, and gravity force. We also present a general thermodynamic analysis of the states and state variables in the monolayer systems. In Sec. III, we present a detailed analysis of the weak undulation case where we only keep terms up to the quadratic orders in the height of the interface and its gradient. We highlight the subtle effects of gravity in determining the wavelength of the undulation and the phase behaviors and the scaling dependence of the critical wavelength on the spontaneous curvature and gravity. We also estimate the range of the weak undulation regime and point out the necessity for studying the full theory. In Sec. IV we present some preliminary results of the nonlinear theory obtained by using a simple variational ansatz. An important result in this section is that the undulated phase disappears if the two components becomes strongly immiscible. Section $\mathrm{V}$ contains a condensed discussion of monolayers at the fluid-solid interface (henceforth referred to as solid-supported monolayers), drawing analogy, and making reference for details to the previous sections. Our analysis throughout this paper is based on mean-field theory though some effects due to fluctuations will also be discussed briefly where appropriate. Section $\mathrm{V}$ concludes the paper with a discussion of the main results and future problems, and the Appendix contains some mathematical details pertaining to the derivation of Eq. (4) in Sec. II.

\section{LIQUID-SUPPORTED MONOLAYERS: FREE ENERGY AND THERMODYNAMIC ANALYSIS}

We consider a smooth, fluid (as opposed to tethered) interfacial film of area $A$ described by the parametric function $\mathbf{R}=\mathbf{R}(\mathbf{s})$, where $\mathbf{s}$ is the (two-dimensional) coordinate on the surface. We assume that the film is incompressible, so that its composition is fully specified by the order parameter $m(\mathbf{s})$ which is a measure of the local deviation from the homogeneous, symmetric mixture. We use a Ginzburg-Landau free energy functional to describe the local variation of this order parameter. To quartic order, this free energy can be written as

$$
\begin{aligned}
F_{c} / k T= & \int_{A} d^{2} \mathbf{s}\left\{(1 / 2) t m(\mathbf{s})^{2}+(1 / 2) b a^{2}\left[\nabla_{s} m(\mathbf{s})\right]^{2}\right. \\
& \left.+(1 / 4) u m(\mathbf{s})^{4}-\mu m(\mathbf{s})\right\},
\end{aligned}
$$

where $a$ is some microscopic length scale (e.g., the nearestneighbor distance between head groups) and $\mu$ is the chemical potential difference between the two components.

To describe the free energy change due to shape fluctuations, we generalize the Helfrich curvature elastic free 
energy for fluid membranes ${ }^{9}$ to a two-component film. To quadratic order in curvatures, this free energy reads

$$
\begin{aligned}
F_{\mathrm{el}} / k T= & \int_{A} d^{2} \mathrm{~s}\left\{2 K\left[H(\mathrm{~s})-H_{0}(\mathrm{~s})\right]^{2}-2 K H_{0}(\mathrm{~s})^{2}\right. \\
& +\bar{K} G(\mathrm{~s})\}
\end{aligned}
$$

where $H(\mathrm{~s})$ is the local mean curvature, $H_{0}(\mathrm{~s})$ is the spontaneous curvature and $G(s)$ is the Gaussian curvature. The term $-2 \mathrm{KH}_{0}(\mathrm{~s})^{2}$ is included to make the flat film the reference state for calculating the free energy. Notice that whereas in a single component membrane $H_{0}$ is a material constant across the membrane, in our case, $H_{0}(\mathrm{~s})$ varies with position through its composition dependence. In this study, we assume that the two amphiphiles have opposite spontaneous radii of curvatures of the same magnitude $R_{0}$ so that to lowest orders we may write $H_{0}(\mathrm{~s})$ as $H_{0}(\mathrm{~s})$ $=m(\mathrm{~s}) / R_{0}$. To leading order, the bending moduli $K$ and $\bar{K}$ are independent of the composition of the film. [One may write $K$ and $\bar{K}$ as expansions in $m$, and the corrections due to the $m$ dependence of these bending moduli are of higher orders than the leading terms. For a discussion of the bending moduli of mixed surfactants, see Ref. 19.] By virtue of the Gauss-Bonnet theorem, the Gaussian curvature term yields a topological constant after integration over the whole surface. Since we will only be concerned with shape changes without change in the topology of the film, we can drop this constant from our free energy expression.

The final free energy contribution arises from capillary waves which incur a cost in the gravitation energy due to undulation of the surface. If the local deviation from the flat horizontal position of the surface is $h(\mathbf{r})$, where $\mathbf{r}$ is the (laboratory) coordinate in the horizontal plane, then this free energy is

$$
F_{g} / k T=(1 / 2)(k T)^{-1} \int_{A_{0}} d^{2} \mathbf{r} \Delta \rho g h(x, y)^{2},
$$

where $\Delta \rho$ is the density difference between water and air (or two other fluids), $g$ is the earth gravity constant, and $A_{0}$ is now the area of the flat base. In writing Eq. (3), we have implicitly assumed that the function $h(x, y)$ is single valued, i.e., there are no overhangs in the location of the surface. We will assume that this is so throughout the paper. In this case, it is more convenient to work with the coordinate system $(x, y)$ and the height of the surface $h(x, y)$ rather than the surface coordinate $\mathbf{s}$ and its threedimensional position $\mathbf{R}$. This transformation is straightforward for all the terms of the free energy except the gradient square term in Eq. (1) whose transformation is derived in the Appendix. We have, after the coordinate transformation,

$$
\begin{aligned}
F / k T= & \int_{A_{0}} d^{2} \mathbf{r}\left[1+(\nabla h)^{2}\right]^{1 / 2}\left((1 / 2) t m^{2}+(1 / 4) u m^{4}-\mu m+(1 / 2) b\left\{(\nabla m)^{2}-(\nabla m \cdot \nabla h)^{2} /\left[1+(\nabla h)^{2}\right]\right\}\right) \\
& +\int_{A_{0}} d^{2} \mathbf{r}\left[1+(\nabla h)^{2}\right]^{1 / 2}\left((1 / 2) K\left\{\left[\nabla^{2} h+(\nabla h)^{2} \nabla^{2} h-\nabla h \nabla h: \nabla \nabla h\right] /\left[1+(\nabla h)^{2}\right]^{3 / 2}\right\}^{2}\right. \\
& \left.-\lambda K m\left[\nabla^{2} h+(\nabla h)^{2} \nabla^{2} h-\nabla h \nabla h: \nabla \nabla h\right] /\left[1+(\nabla h)^{2}\right]^{3 / 2}\right)+\int_{A_{0}} d^{2} \mathbf{r}(1 / 2) \zeta h^{2},
\end{aligned}
$$

where we have scaled all lengths, including the height of the interface, by the microscopic length scale $a$, and the dimensionless parameters are $\lambda=2 a / R_{0}$ and $\xi=\Delta \rho g a^{4} /$ $k T$. $\zeta$ can be written more conveniently if we define the capillary length $\Lambda_{c}=(\Delta \rho g / k T)^{1 / 4}$; then $\zeta$ is simply $\xi=\left(a / \Lambda_{c}\right)^{4}$. The parameters $b$ and $u$ can be estimated from the regular solution theory; quantitative estimates will be discussed later.

To obtain the equilibrium profile for $m(\mathbf{r})$ and $h(\mathbf{r})$ for a fixed amount of surface area, we minimize Eq. (4) subject to the constraint

$$
A\{[h(\mathbf{r})]\}=\int_{A_{0}} d^{2} \mathbf{r}\left[1+(\nabla h)^{2}\right]^{1 / 2}
$$

This can be achieved by introducing a Lagrange multiplier $\eta$ and minimizing the new thermodynamic function

$$
G / k T=F / k T-\eta \int_{A_{0}} d^{2} \mathbf{r}\left[1+(\nabla h)^{2}\right]^{1 / 2}
$$

It is convenient to regard $G$ as the grand potential for an open system where the amount of both components, or equivalently, the total amount of interface and the composition (controlled by $\mu$ ) can vary freely. This view of $G$ leads naturally to the definition of a surface pressure (tension) $\pi$ such that $G=-\pi A_{0} \cdot{ }^{20} \pi>0$ corresponds to compression of the film, whereas $\pi<0$ corresponds to tensile extension. For a compressed single-component film, Milner et al. ${ }^{21}$ have shown that a buckling instability occurs at some critical compression pressure with a wave number $q_{c}=K^{1 / 4} / \Lambda_{c}$. The physical reason for such a buckling instability is clear: in order to accommodate excess amount of interface on a flat base, the interface has to wrinkle. Similar buckling instabilities are also expected for our mixed surfactant film when it is under compression. In a 
sense, the phenomena of buckling under compression is "trivial" as this arises merely from the need to accommodate the excess amount of interface. The more interesting case is when the film is free of tension or compression. In this case, $G=0$. It can be shown that this condition is equivalent to minimizing the free energy per unit area (of the fluctuating interface) $F / A$, where $A$ is given by $\mathrm{Eq}$. (5). This is the point of adsorption saturation, ${ }^{22,23}$ whereby the surfactant film coexists with surfactants in solution and is the situation which we focus in this paper. For this case, the minimization conditions become

$$
\begin{aligned}
& \delta F\{[m(\mathbf{r}), h(\mathbf{r})]\} / \delta m(\mathbf{r})=0, \\
& \delta F\{[m(\mathbf{r}), h(\mathbf{r})]\} / \delta h(\mathbf{r})=\eta \delta A\{[h(\mathbf{r})]\} / \delta h(\mathbf{r}),
\end{aligned}
$$

and

$$
\eta=F / A \text {. }
$$

\section{LIQUID-SUPPORTED MONOLAYERS: RIPPLING TRANSITION IN THE NEAR-CRITICAL REGION}

To study the onset of rippling and the scaling of the critical wavelength, we keep terms only up to $O\left[h^{2},(\nabla h)^{2},\left(\nabla^{2} h\right)^{2}\right]$. The free energy now becomes

$$
\begin{aligned}
F\{[m(\mathbf{r}), h(\mathbf{r})]\}= & \int_{A_{0}} d^{2} \mathbf{r}\left[(1 / 2) t m^{2}+(1 / 2) b(\nabla m)^{2}\right. \\
& \left.+(1 / 4) u m^{4}-\mu m\right] \\
& +\int_{A_{0}} d^{2} \mathbf{r}\left[(1 / 2) K\left(\nabla^{2} h\right)^{2}\right. \\
& \left.-\lambda K m \nabla^{2} h+(1 / 2) \xi h^{2}\right]
\end{aligned}
$$

and the area $A$ is given approximately by

$$
A\{[h(\mathbf{r})]\}=\int_{A_{0}} d^{2} \mathbf{r}\left[1+(1 / 2)(\nabla h)^{2}\right] .
$$

Notice that in Eq. (8), we have ignored such terms as $m^{2}(\nabla h)^{2},(\nabla m)^{2}(\nabla h)^{2}$, etc. It could be argued that to be completely consistent, these terms should be included. However, they are not expected to change the smallamplitude behaviors in any qualitative way. For locating the transition point, only the quadratic coefficients count and these higher order terms can be justifiably ignored.

For small amplitude undulations, it can be seen from Eq. (7c) that $\eta$ is of the order of $O\left[h^{2},(\nabla h)^{2},\left(\nabla^{2} h\right)^{2}\right]$. Thus to the quadratic order, the right-hand side of Eq. (7b) can be set to zero as it will be of higher orders. Thus we may regard $\eta$ as zero, and Eqs. (7a) and (7b) become an unconstrained variational problcm.

At the quadratic order, minimization of $F$ with respect to $h$ is easily accomplished by introducing the Fourier transforms

$$
m_{\mathbf{k}}=\left(1 / A_{0}\right) \int_{A_{0}} d^{2} \mathbf{r} m(\mathbf{r}) \exp (-i \mathbf{k} \cdot \mathbf{r})
$$

and

$$
h_{\mathbf{k}}=\left(1 / A_{0}\right) \int_{A_{0}} d^{2} \mathbf{r} h(\mathbf{r}) \exp (-i \mathbf{k} \cdot \mathbf{r})
$$

and we find

$$
h_{\mathrm{k}}=i \lambda K k^{2}\left(K k^{4}+\zeta\right)^{-1} m_{\mathrm{k}} .
$$

Thus we see that a spatial modulation in $m$ gives rise to an undulation in $h$ and vice versa. Substituting Eq. (10) in the free energy leads to the following effective free energy in terms of $m_{\mathrm{k}}$ :

$$
\begin{aligned}
F\left(m_{\mathrm{k}}\right)= & (1 / 2) A_{0} \sum_{\mathbf{k}} Q_{\mathrm{k}} m_{\mathrm{k}} m_{-\mathrm{k}}-A_{0} \mu m_{0} \\
& + \text { higher order terms, }
\end{aligned}
$$

where

$$
Q_{\mathrm{k}}=t+b k^{2}-\lambda^{2} K^{2} k^{4} /\left(K k^{4}+\zeta\right) .
$$

A spatially modulated phase first appears when $Q_{\mathrm{k}}$ becomes negative for some nonzero $k$. This leads to the following inequality for the parameters in the theory:

$$
\lambda^{4}>4 b^{2} K^{-3} \xi
$$

which is met by most surfactant systems. (A more quantitative estimate will be discussed later.) Therefore, with an appropriate choice of a binary mixture, this rippling instability should be easy to observe.

Next, we look for the critical wave number of the undulation. This corresponds to the minimum of $Q_{\mathrm{k}}$ [we will assume hereafter that the inequality Eq. (13) is always satisfied]. Setting $\partial Q_{\mathrm{k}} / \partial k^{2}=0$, we have

$$
\sqrt{2} \lambda K \xi^{-1 / 2} k=b^{1 / 2}+b^{1 / 2} K \xi^{-1} k^{4} \text {. }
$$

It is convenient to define $\sigma=4 b^{2} \zeta /\left(\lambda^{4} K^{3}\right)$; evidently $0<\sigma<1$ [cf. Eq. (13)]. We seek to bring Eq. (14) into the scaling form

$$
2(k / \kappa)=\sigma^{\nu}+(k / \kappa)^{4},
$$

where we have introduced a wave number scale $\kappa$. Comparing Eq. (15) with Eq. (14), along with the definition of $\sigma$, we find

$$
\kappa=2^{-1 / 6} b^{-1 / 6} \lambda^{1 / 3} \xi^{1 / 6}
$$

and $v=1 / 3$. Thus the critical wave number $k_{c}$ is

$$
k_{c}=\kappa \phi(\sigma) \text {, }
$$

where the function $\phi$ is the real, positive solution to $2 \phi$ $=\sigma^{1 / 3}+\phi^{4}$, with $\phi(0)=2^{1 / 3}$ and $\phi(1)=1$.

At the critical wave number,

$$
Q\left(k_{c}\right)=-(1 / 2) \lambda^{2} K \phi(\sigma)^{2}\left[b^{-1} \phi(\sigma)-\sigma^{1 / 3}\right] \equiv-g_{0}
$$

Thus we have an upward shift of the effective critical temperature by an amount of $g_{0}$. In the critical region, the free energy takes the form of a Brazovskii model, ${ }^{24}$ which has been studied in some detail by Garel and Doniach ${ }^{25}$ in the context of uniaxial magnetic film and by Andelman et al. ${ }^{26}$ in the context of a Langmuir monolayer of dipolar molecules. In particular, Andelman et al. have calculated the $t-m$ phase diagram near the critical point and have found 
five possible phases-a uniform $m>0$ phase, a hexagonally ordered droplet phase with the droplet region having $m<0$, a stripe phase with alternating regions of $m<0$ and $m>0$, a hexagonally ordered droplet phase with the droplet region having $m>0$, and a uniform phase with $m<0$. Except for some possible complications due to higher order coupling terms such as $m^{2}(\nabla h)^{2}$, we expect a similar phase diagram for the case studied here with accompanying undulations (in the case of spatially modulated phases) in the surface height profile. Far below the critical temperature, of course, our free energy differs from the Brazovskii model.

It is interesting to note the peculiar dependence of the critical wavelength on the spontaneous radius of curvature and on the capillary length. Since $\phi$ is a very weak function of $\sigma$, the dominant dependence of $k_{c}$ on $\lambda$ and $\zeta$ is contained in the power-law scaling dependence [Eq. (16)]. We will see next that for most surfactant systems, we expect $\sigma \ll 1$, thus $Q\left(k_{c}\right)$ is practically $-(1 / 2) b^{-1} \lambda^{2} K \phi(0)^{3}$ $=-\lambda^{2} K b^{-1}$, which is independent of the capillary length. Thus, while gravity has a significant effect on the critical wavelength of undulation, it has little effect on the phase diagram in the near-critical region.

At this point, it is helpful to have a quantitative estimate of the parameters appearing in the theory. We take our microscopic length scale to be $a=5 \AA$. For typical short-chain surfactants, $R_{0} \approx 50 \AA$ and $K \approx 2$ (in units of $\mathrm{kT}) \cdot{ }^{27}$ With $\Delta \rho \approx 1 \mathrm{~g} /(\mathrm{cm})^{3}$ (between water and air), the capillary length at room temperature is roughly $8000 \AA$. To estimate the parameters $b$ and $u$ appearing in Eq. (1), we resort to a lattice regular solution model in two dimension (Bragg-Williams solution of the two-dimensional binary mixture model). In this way, we obtain $b=1 /(2 d)$ $=1 / 4$ and $u=1 / 3$. Using these parameters, we estimate the critical wavelength $L_{c}=2 \pi / k_{c}$ to be roughly $5196 \AA$ which should be within the accessible range of typical optical experiments.

It is also of interest to estimate the validity of the critical (small amplitude) region. In order for our preceding analysis to be valid, we must have $(\nabla h)^{2} \ll 1$ or $k_{c}^{2} h_{\mathrm{k}} h_{-\mathrm{k}} \ll 1$. It can be shown with the aid of Eq. (10) that

$$
k_{c}^{2} h_{\mathrm{k}} h_{-\mathrm{k}}=2^{-1 / 3} b^{1 / 3}\left(\Lambda_{c} / R_{0}\right)^{4 / 3} m_{\mathrm{k}} m_{-\mathrm{k}} .
$$

Just below the critical temperature, the spatial modulation is dominated by a sinusoidal wave with the critical wavelength. For a symmetric composition $(\mu=0)$, the amplitude of the one-dimensional wave is found to be

$$
m_{\mathrm{k}} m_{-\mathrm{k}}=(4 / 3) u^{-1}\left(g_{0}-t\right)
$$

[in obtaining this result, we have ignored terms such as $m^{2}(\nabla h)^{2}$ ]. If we consider $k_{c}^{2} h_{\mathrm{k}} h_{-\mathrm{k}}=1$ to be the limit of validity of the small amplitude approximation, we can determine the range for the effective temperature $t_{e}=t-g_{0}$, where the approximation is valid. Combining Eqs. (19) and (20), and setting $k_{c}^{2} h_{\mathrm{k}} h_{-\mathrm{k}}=1$, we can see that the small amplitude approximation is valid only when

$$
-(3 / 4) 2^{1 / 3} b^{-1 / 3} u\left(R_{0} / \Lambda_{c}\right)^{4 / 3}<t_{e}<0 .
$$

For $R_{0}=50 \AA$, the left-hand side of Eq. (21) is -5.8 $\times 10^{-4}$, a very small range indeed. Therefore, for short surfactant systems, experimentally it is difficult to observe the small amplitude region. Possible systems for which this region is observable are diblock copolymers for which the spontaneous radius of curvature can be very large. If we take $R_{0}=500 \AA$, the left-hand side of Eq. (21) becomes $-1.2 \times 10^{-2}$.

\section{LIQUID-SUPPORTED MONOLAYERS: LOW TEMPERATURE PHASES}

Physically, the small-amplitude region analyzed in the previous section corresponds to slightly incompatible surfactant mixtures. The incompatibility has to be just large enough for $Q_{\mathrm{k}}$ to be negative at the critical wavelength, but not too negative. It is clear from the previous section that this regime is in a very narrow temperature range. For more incompatible mixtures, we cannot treat $\nabla h$ as being small and therefore have to use the full nonlinear form (4). A general treatment using Eq. (4) is necessarily rather complicated, so we limit our discussion to the symmetric mixture $(\mu-0)$, where the rippled phase is expected to be a one-dimensional undulation. However, some of the conclusions drawn for this special case are also expected to be valid for general situations.

Let us analyze the physical factors affecting the rippling of the interface at low temperatures (by which we mean strongly incompatible mixtures). Clearly, the driving force for the rippling of the interface is the opposite spontaneous curvatures of the two components of the monolayer. By arranging the two components in an alternating fashion, each component achieves a certain satisfaction of its spontaneous curvature. However, this tendency for rippling is countered by two opposing factors. First, gravity keeps the interface from making large amplitude undulations, and second, the periodic arrangement of the two (incompatible) components introduces domain wall free energy cost, which increases with decreasing temperatures and/or wavelength. In the near critical region, as the temperature (i.e., $t_{e}$ ) decreases from $t_{e}=0$, the wavelength should first decrease; this is because the spontaneous curvature of the mixture is $\lambda m$ and the magnitude of $m$ increases as temperature decreases. Thus the effective spontaneous curvature of the mixture increases and hence the wavelength of undulation decreases. For very low temperatures, $m$ is expected to reach a saturated value $(\approx \pm 1)$, sharp boundaries develop between domains and the orderparameter expansion (1) is no longer a good approach. In this regime, the gain in the spontaneous curvature energy will reach a saturated value and this value has to compete with the cost incurred by the formation of sharp domain walls and the penalty due to gravity. It is this competition between the spontaneous curvature energy on the one hand and the domain-wall energy and gravitation energy on the other that determines the wavelength of the undulation and indeed determines if the undulated phase is favorable at all. The result of this competition may eventually make it so unfavorable energetically to have spatial modulation that the system prefers macroscopic phase separation into 
two bulk phases without undulation in the interfacial profile. It is likely that this balance occurs even earlier at some finite wavelength, in which case, we expect a first order transition to the uniform phases.

To make the above argument more quantitative, we have carried out a variational calculation. For a onedimensional wave, it is expected that the undulation consists of alternating domains of the two surfactant species whose composition is nearly uniform with $m$ reaching the saturated value \pm 1 and which are separated by narrow domain walls with a wall energy (per unit length) $\gamma$. Taking one-half of a period with say $m=1$, we make a variational ansatz for the height of the interfacial profile, assuming the undulation is in the $x$ direction

$$
h=(1 / 2) \alpha\left(D^{2}-x^{2}\right)(-D \leqslant x \leqslant D),
$$

where $D$ is one-quarter of the period, and for convenience, we have set the origin at the maximum height of the profile. Then the three dominant free energy contributions are evaluated to be

$$
\begin{aligned}
F_{\mathrm{el}}= & \left\{K \alpha^{2} D\left[1+(2 / 3) \alpha^{2} D^{2}\right] /\left(1+\alpha^{2} D^{2}\right)^{3 / 2}\right. \\
& -2 K \lambda \arctan (\alpha D)\} L, \\
F_{g}= & {\left[(2 / 15) \xi \alpha^{2} D^{5}\right] L, } \\
F_{w}= & \gamma L,
\end{aligned}
$$

for the curvature elastic energy, the gravity energy, and the wall energy, respectively. $L$ is the length of the system in the $y$ direction. The area of the half-period is

$$
\begin{aligned}
A= & L D\left\{\left(1+\alpha^{2} D^{2}\right)^{1 / 2}\right. \\
& \left.+\alpha^{-1} D^{-1} \ln \left[\alpha D+\left(1+\alpha^{2} D^{2}\right)^{1 / 2}\right]\right\} .
\end{aligned}
$$

The free energy per unit area is then $f=\left(F_{\mathrm{el}}+F_{g}+F_{w}\right) / A$. We first minimize $f$ over $\alpha$ to obtain a free energy as a function of $D$. This free energy for three different values of $\gamma$ 's is plotted in Fig. 2, where $f_{0}$ in the ordinate is the free energy per unit area for the uniform phase without undulation under the same conditions (i.e., $m=1, \alpha=0$, and $1 / D=0$ ). The other parameters used in the plot are $K=2$, $R_{0}=50 \AA(\lambda=0.2)$, and $\Lambda_{c}=8000 \AA$. It is readily seen that the wavelength of the undulation increases as the wall energy increases. For $\gamma=1.2$, the free energy is positive for nonzero $a / D$; thus at this value of $\gamma$, we expect that the system will prefer a macroscopically separated uniform phase without undulation. Figure 2(b) shows a magnified view of the free energy for the long wavelength regions. The local maxima at $a / D>0$ suggest that the transition from spatially modulated stripes to a uniform phase is likely to be first order. Note, however, the small scale of the free energy difference in the plot. With such shallow free energy minima, the fluctuations in the domain sizes are expected to be very large. Instead of well-ordered stripes, experimentally we may see domains of irregular shapes which could resemble a two-dimensional microemulsion pattern.

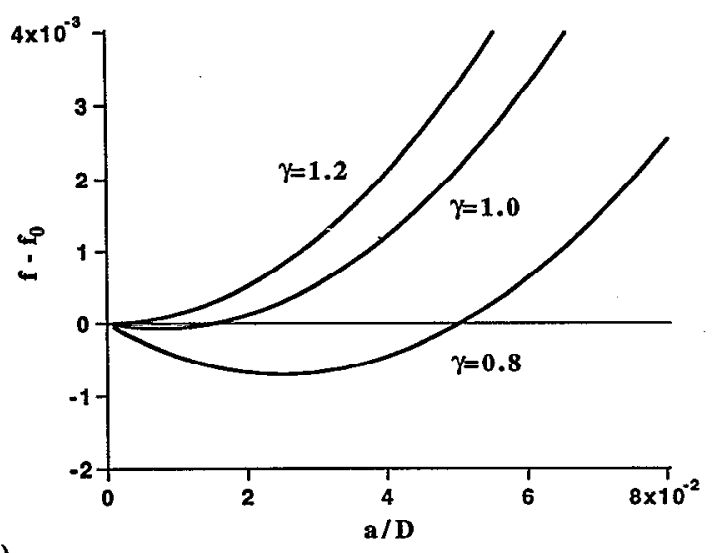

(a)

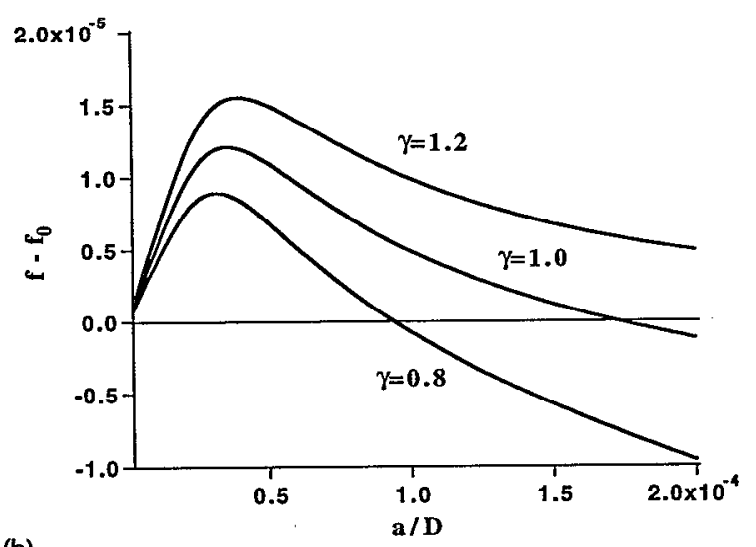

(b)

FIG. 2. Free energy per unit area as a function of the periodicity for a liquid-supported binary surfactant monolayer. Here $D$ is $1 / 4$ of the period and $a$ is the microscopic length scale. (b) is a magnified view of (a) in the small $a / D$ region. The reference free energy $f_{0}$ is taken to be that of a uniform, macroscopically separated phase.

\section{SOLID-SUPPORTED MONOLAYERS}

We consider a film of constant surface density, so the free energy associated with the composition variation can again be described by a Ginzburg-Landau free energy expansion in the order parameter $m$. Because the surface is always flat, the surface coordinate coincides with the laboratory flat base coordinates and we can write the free energy as

$$
\begin{aligned}
F_{c} / k T= & \int_{A_{0}} d^{2} \mathbf{r}\left[(1 / 2) t m^{2}+(1 / 2) b(\nabla m)^{2}\right. \\
& \left.+(1 / 4) u m^{4}-\mu m\right],
\end{aligned}
$$

where, again, the lengths are all scaled by some microscopic length scale $a$. We use a unit vector $\hat{n}$ to describe the orientation of the surfactant. For the free energy of director fluctuations, we assume that the mixture system is globally in an untilted phase (the analog of the $3 d$ smectic- $A$ phase), so that there is no preferred tilt angle. This feature is captured by a term $(1 / 2) s c \cdot c$ which penalizes the unit director $\hat{n}$ for having components parallel to the surface $\mathbf{c}=\hat{n}-\hat{z}(\hat{z} \cdot \hat{n})$. Alternatively, this term can be thought of as the lowest order, nontrivial term in the expansion of $-s \hat{z} \cdot \hat{n}$ which is permitted because of the lack of 
inversion symmetry (with respect to the anchoring surface) in the system and physically favors the upward orientation. In analogy to $3 D$ liquid crystals, ${ }^{28}$ we also include Frank elastic energy terms for splay $(1 / 2) K_{s}(\nabla \cdot \hat{n})^{2}$ $=(1 / 2) K_{s}(\nabla \cdot \mathbf{c})^{2}$ and (in-plane) bend $(1 / 2) K_{b}(\nabla \times \mathbf{c})^{2}$ $=(1 / 2) K_{b}[(\hat{z} \times \nabla) \cdot c]^{2}$. The special feature alluded to throughout the paper, namely, the head-tail asymmetry, amounts to a spontaneous splay term which is proportional to the local composition inhomogeneity $m,-K_{s} \lambda_{s} m \nabla \cdot \hat{n}$ $=-K_{s} \lambda_{s} m \nabla \cdot c$. Thus the elastic part of the free energy is

$$
\begin{aligned}
F_{\mathrm{el}} / k T= & \int_{A_{0}} d^{2} \mathbf{r}(1 / 2) s c^{2}+(1 / 2) K_{s}(\nabla \cdot \mathbf{c})^{2} \\
& +(1 / 2) K_{b}(\nabla \times \mathbf{c})^{2}-K_{s} \lambda_{s} m \nabla \cdot \mathbf{c} .
\end{aligned}
$$

(The reason for writing the coefficient of the spontaneous splay term as $K_{s} \lambda_{s}$ will become clear later when we compare solid-supported monolayers with liquid-supported monolayers.) The total free energy is simply the sum of Eqs. (25) and (26).

The composition and director profiles are obtained in a straightforward manner by minimizing the free energy with respect to these order parameters. Note here that the amount of surfactants is fixed once we specify the area of the interface and assume incompressibility of the film. Thus the complications encountered in the liquidsupported film where the amount of surfactants varies with the interfacial height profile do not arise.

Because the free energy is quadratic in c, it is most convenient to first minimize the free energy with respect to c by using a Fourier transform; this gives

$$
\mathbf{k} \cdot \mathbf{c}_{\mathbf{k}}=i K_{s} \lambda_{s} k^{2} m_{\mathbf{k}} /\left(s+K_{s} k^{2}\right)
$$

and

$$
(\hat{z} \times \mathbf{k}) \cdot \mathbf{c}=0 .
$$

Substituting Eq. (27) into the full free energy, we obtain an effective free energy for the order parameter $m$. In Fourier modes, this free energy is

$$
\begin{aligned}
F / k T= & A_{0} \sum_{\mathrm{k}}(1 / 2) Q_{\mathrm{k}} m_{\mathrm{k}} m_{-\mathrm{k}}-A_{0} \mu m_{0} \\
& + \text { higher order terms }
\end{aligned}
$$

with

$$
Q_{\mathrm{k}}=t+b k^{2}-K_{s}^{2} \lambda_{s}^{2} k^{2} /\left(s+K_{s} k^{2}\right) .
$$

The critical point is located at $\partial Q_{\mathrm{k}} / \partial k^{2}=0$ and $Q_{\mathrm{k}}=0$, which yield

$$
k_{c}=\left(s / K_{s}\right)^{-1 / 2}\left(K_{s} \lambda_{s} b^{-1 / 2} s^{-1 / 2}-1\right)^{1 / 2}
$$

for the critical wave vector and

$$
t_{c}=K_{s} \lambda_{s}^{2}\left(1-b^{1 / 2} s^{1 / 2} K_{s}^{-1} \lambda_{s}^{-1}\right)^{2}
$$

for the critical temperature. In order to have a nonzero $k_{c}$, we must have $K_{s} \lambda_{s}>b^{1 / 2} s^{1 / 2}$.

In order to have an estimate for the values of the elastic constants $K_{s}$ and $\lambda_{s}$, it is instructive to relate them to the curvature elastic constants appearing in the free energy

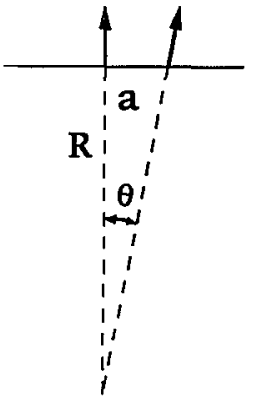

FIG. 3. An illustration of the relationship between a splay deformation of the molecular orientation in the solid-supported film and a curvature deformation in the corresponding liquid-supported film.

for the liquid-supported monolayer. Such a relationship should exist because curvature elasticity and director elasticity have a common physical origin. On a local scale, splay of the director has the same effect as curving the interface, namely, to change the tail-tail distance. ${ }^{9}$ The curl deformation of the director is more subtle, but since it does not appear in our (mean-field) free energy, we focus on $K_{s}$ and $\lambda_{s}$. Consider for simplicity a onc-dimensional splay. Now $\nabla \cdot c \approx(1 / a)\left[c_{x}(x+a)-c_{x}(x)\right]$, where $a$ is some microscopic length scale (e.g., nearest-neighbor head-head distance) and $c_{x}$ is the $x$ component of the unit director $\hat{n}$. For a small splay, $c_{x}(x+a)-c_{x}(x) \approx \theta$, where $\theta$ is the angle between the two directors. On the other hand, from simple geometry as illustrated in Fig. 3, $a \approx \theta R$, where $R$ is the (extrapolated) radius. Putting these pieces together, we have $\partial c_{x} / \partial x \approx 1 / R$. Thus $K_{s}$ corresponds to the curvature bending constant $K$ for the liquid-supported film and the spontaneous splay corresponds to the spontaneous curvature. Hence, as a zeroth-order approximation, we can write $\lambda_{s}$ as $\lambda_{s}=2 a / R_{0}$, where $R_{0}$ can be thought of as the spontaneous radius of curvature of the corresponding liquid-supported monolayer. Since typically $R_{0} \sim 50 \AA$, we expect $\lambda_{s} \sim 0.2$. On the other hand, the solid nature of the substrate can make $K_{s}$ significantly different from its liquid-supported monolayer counterpart.

The free energy (28) has the form of the Brazovskii model. Therefore, we expect the phase diagram near the order-disorder transition to be similar to the ones studied by previous authors. As a function of the composition, we expect to see stripe phases for symmetric compositions and hexagonally ordered "bubble" phases for asymmetric compositions with the minority species forming the bubbles.

At very low temperatures, the order-parameter expansion (25) for small $m$ is no longer appropriate. The general analysis for low temperatures is difficult, so we again specialize to the one-dimensional wave with the symmetric composition (i.e., $\mu=0$ ). We assume, as we did for the fluid-fluid film, that the two components separate into alternating, distinct domains with sharp domain walls. Each domain is occupied exclusively by one of the components $(m \approx \pm 1)$. Thus the free energy can once again be separated into an elastic contribution and a domain wall contribution. Consider half of a period occupied by one of the 


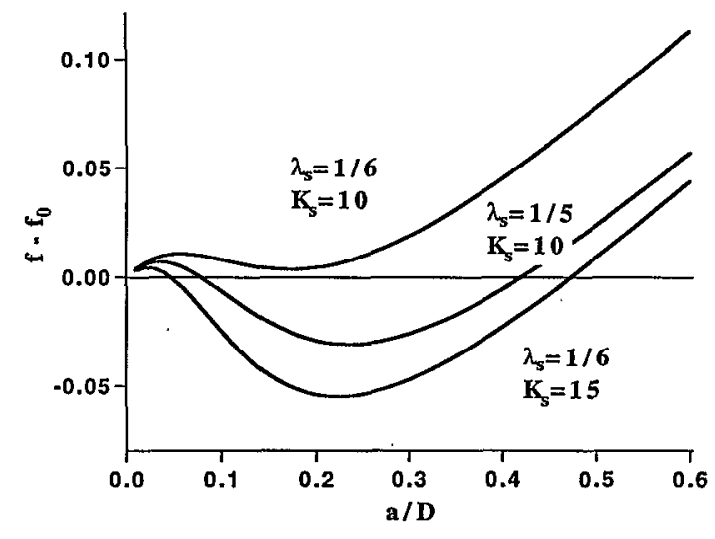

FIG. 4. Free energy per unit area as a function of the periodicity for a solid-supported binary surfactant monolayer. The reference free energy $f_{0}$ is taken to be that of a uniform, macroscopically separated phase.

species characterized by a positive spontaneous splay. We make the variational ansatz that the director field $c$ (as measured by the projection of the molecular director to the direction of the wave) varies linearly within the domain, i.e., $c=c_{0}(x / D)$ for $-D \leqslant x \leqslant D$, where $D$ is a quarter of the period; then the free energy per unit area becomes

$$
\begin{aligned}
f-f_{0}= & (1 / 6) s c_{0}^{2}+(1 / 2) K_{s} c_{0}^{2} / D^{2}-K_{s} \lambda_{s} c_{0} / D \\
& +(1 / 2) \gamma / D,
\end{aligned}
$$

where $f_{0}$ is the free energy of the uniform, macroscopically separated phase $(m=1, c=0$, and $1 / D=0)$. We first minimize $f$ with respect to $c_{0}$, thus obtaining the following free energy as a function of $D$ :

$$
\begin{aligned}
f-f_{0}= & -(1 / 2) K_{s}^{2} \lambda_{s}^{2} D^{-2} /\left[(1 / 3) s+K_{s} / D^{2}\right] \\
& +(1 / 2) \gamma / D .
\end{aligned}
$$

This free energy is plotted in Fig. 4 for $s=1, \gamma=0.8$ and different values of the spontaneous splay $\lambda_{s}$ and the elastic constant $K_{s}$. The three curves in the figure are intended to show the effects of both the spontaneous splay (measured by $\lambda_{s}$ ) and the elastic (stiffness) constant $K_{s}$. As expected on physical grounds, for a given $K_{s}$, large $\lambda_{s}$ favors modulated phases because the gain in spontaneous splay energy has to be large enough to overcome the wall energy cost. Similarly, for a given $\lambda_{s}$, a larger $K_{s}$ makes a larger gain in the spontaneous splay energy and hence favors spatially modulated phases. Although not shown in the figure, we expect a large wall energy to disfavor spatially modulated phases. When the gain in the spontaneous splay energy can no longer compensate for the cost incurred by the domain wall energy, the system chooses to separate macroscopically into two (2D) bulk phases. Again, it is interesting to note that the free energy function has the feature of a first-order transition in going between the spatially modulated phase and the macroscopically separated uniform phase, as the parameters $\left(\lambda_{s}, K_{s}\right.$ and $\left.\gamma\right)$ are varied; this implies that the transition from the stripe phase to the uniform phases occurs at some finite wavelength. Given the simple form of the free energy (32), we can locate the point of the transition from the stripe phase to the uniform phase; this is found from $f-f_{0}=0$ and $\partial\left(f-f_{0}\right) / \partial D=0$ whence we obtain

$$
\gamma=(1 / 2) K_{s} \lambda_{s}^{2}\left(3 K_{s} / s\right)^{1 / 2}
$$

Thus we have the striped phase for $\gamma<(1 / 2) K_{s} \lambda_{s}^{2}$ $\times\left(3 K_{s} / s\right)^{1 / 2}$ and the macroscopically separated, uniform phase for $\gamma>(1 / 2) K_{s} \lambda_{s}^{2}\left(3 K_{s} / s\right)^{1 / 2}$. Notice that the free energy scale for the minima and barrier is much larger than in the corresponding figure for the liquid-supported monolayer. Thus the low temperature stripes can be quite stable and the first-order nature in the transition between the stripe phase and the uniform phase can be fairly pronounced.

\section{CONCLUSIONS}

In this paper, we have focused on an important aspect of surfactant monolayers, namely, the head-tail asymmetry and its consequences in the structures and phase transitions. Although this head-tail asymmetry has been known to have important effects in determining the shapes of micellar aggregates and microemulsion droplets, considerably fewer studies have been conducted which address its effects in the monolayer systems. This relative lack of attention is probably because most in-depth structural studies of monolayers have focused on single-component systems, and in these single component systems, the effects of spontaneous curvature or the spontaneous splay are suppressed for the reasons mentioned in the Introduction. However, in binary mixtures, these effects can have manifest consequences. Our study in this paper provides a phenomenological account for the structures and phase transitions that can take place in a monolayer of binary surfactant mixtures when the head-tail asymmetry becomes important. Specifically, our study shows that local composition variation, when coupled to the spontaneous curvature or splay, can lead to thermodynamically stable, spatially modulated phases. Estimates using reasonable values for the parameters in our theory suggest that the predicted phenomena should not be difficult to observe experimentally.

The rippling transition of mixed surfactant layers at the fluid-fluid interface has recently been studied by Andelman et al. ${ }^{29}$ using a free energy functional which keeps only up to the quadratic order in $\nabla h$. However, in that work, the effects of gravity were not considered. Rather, it has a surface tension term which penalizes the increase of surface area due to rippling. Their system thus corresponds more to an insoluble monolayer where the amount of surfactants in the monolayer is fixed, but the total area of the monolayer is not. [However, before the saturation of adsorption is reached, the situation can be complicated due to the finite (two-dimensional) compressibility of the film, and variations in the total surfactant density may have to be considered.] Our work focuses on the situation where the monolayer has reached saturated adsorption and therefore there is no net surface tension ${ }^{22,23}$ and the gravity effect becomes the only opposing force for the wrinkling of 
the interface. This difference results in very different behaviors in the fluctuation spectrum of the order parameters as well as different wavelengths for the undulation.

It is interesting to contrast the behavior between monolayers and bilayers of binary surfactant mixtures. In the case of a bilayer, the two components which are of opposite spontaneous curvatures can migrate to the two monolayers comprising the bilayer. By an uneven distribution of the two components between the two monolayers, the system (bilayer) achieves a minimum free energy state with a finite curvature. ${ }^{17}$ In a sense, the two monolayers are two phases each rich in one of the surfactant species; however, by going to different sides of the bilayer, the two phases avoid the usual interfacial region. Thus, there is no domain wall energy cost as in the monolayer cases. MacKintosh and Safran ${ }^{18}$ have further studied the phase diagram for binary surfactant bilayers. In this context, our work studies a complementary situation where the two components are forced to stay in the same monolayer.

In this work, we have treated the film as a fluid film without regard to any spatial order in the head group positions. However, many researchers believe that in the condensed phase, the surfactant molecules have quasilongrange hexatic order. ${ }^{30,31}$ When in addition the molecules are in the tilted phases, the coupling between molecular tilt and the underlying hexatic order can also lead to spatial modulation in the orientation of the molecules. ${ }^{32,33}$ Our paper treats situations where there is no preferred tilt. Furthermore, we have not included any electrostatic interactions which can also cause spatial modulations. ${ }^{26}$ In reality, all these factors can become significant, but nevertheless, the effect of head-tail asymmetry is an important one, especially in binary mixtures.

The approach in this paper is phenomenological and the theoretical analysis is mean field. Thus two natural future directions for the present work are to relate the parameters in the phenomenological theory to molecular parameters and to address thermal fluctuation effects. Interactions involving surfactant molecules are very complex in general, and a real molecular theory for calculating the phenomenological parameters would be a formidable task. Therefore, we will be content with cstablishing some qualitative or semiquantitative relationship between these parameters and some general characteristics of the surfactant molecules, such as the length of the hydrocarbon tail, the size of the head group, etc. Several theoretical calculations have been made on the curvature elastic properties of polymeric surfactant films. ${ }^{19,34}$ Furthermore, Safran et al. ${ }^{35}$ have derived a Ginzburg-Landau free energy for grafted rod-like surfactants interacting via a Lennard-Jones potential. More recently, Kaganer et al. ${ }^{36}$ have studied the tilting transitions of rod-like surfactant monolayers in conjunction with the local positional order. Similar approaches to these works will be followed.

The effects of thermal fluctuations can be addressed at two levels. First, in the critical region, our models are of the Brazovskii type, ${ }^{24}$ therefore, we expect that fluctuations around the critical wave vector will modify the transition to the ordered phases to become weakly first order. Second, two-dimensional striped phases cannot have true long range order because of the Landau-Peierls instability. ${ }^{37}$ The stripe order can only persist within cybotactic groups whose shapes are generally anisotropic. ${ }^{28,38}$ While these fluctuation effects need to be incorporated for an accurate description of the physical systems, our mean-field study is nevertheless useful in elucidating the phenomenology associated with the spontaneous curvature or spontaneous splay effects in monolayers of binary surfactant mixtures.

\section{ACKNOWLEDGMENTS}

This paper is dedicated, with great respect and deep appreciation, to Professor Stuart A. Rice in honor of his 60th birthday. This work has been supported in part by the Camille and Henry Dreyfus New Faculty Award Program and by the Caltech Consortium for Chemistry and Chemical Engineering; founding members E. I. Du Pont de Menours and Company, Inc., Eastman Kodak Company, and Minnesota Mining and Manufacturing Company.

\section{APPENDIX}

In this appendix, we derive the transformation for the term $\left[\nabla_{s} m(\mathbf{s})\right]^{2}$ in Eq. (1) to an expression in terms of the flat-base coordinate $x, y$ and the interfacial profile $h(x, y)$. Take an infinitesimal element of the surface and define a local orthogonal coordinate system $u, v, w$, where $u$ and $v$ are defined along the tangential plane and $w$ is the coordinate along the surface normal. Then,

$$
\begin{aligned}
{\left[\nabla_{\mathrm{s}} m(\mathrm{~s})\right]^{2}=} & m_{u}^{2}+m_{v}^{2} \\
= & m_{x}^{2}\left(x_{u}^{2}+x_{v}^{2}\right)+m_{y}^{2}\left(y_{u}^{2}+y_{v}^{2}\right) \\
& +2 m_{x} m_{y}\left(x_{u} y_{u}+x_{v} y_{v}\right),
\end{aligned}
$$

where $m_{u} \equiv \partial m / \partial u, m_{x} \equiv \partial m / \partial x, x_{u} \equiv \partial x / \partial u$, etc. Any differential vector $d$ s on the surface can be written as

$$
d \mathbf{s}=\hat{u} d u+\hat{v} d v=\hat{x} d x+\hat{y} d y+\hat{z}\left(h_{x} d x+h_{y} d y\right),
$$

where in writing the last term we have made use of $d z$ $=\nabla h \cdot d \mathbf{r}=h_{x} d x+h_{y} d y$.

Taking the dot product of Eq. (A2) with $\hat{x}$ and $\hat{y}$, respectively, yields

$$
\begin{aligned}
& d x=(\hat{x} \cdot \hat{u}) d u+(\hat{x} \cdot \hat{v}) d v, \\
& d y=(\hat{y} \cdot \hat{u}) d u+(\hat{y} \cdot \hat{v}) d v .
\end{aligned}
$$

Thus, we have $x_{u}=\hat{x} \cdot \hat{u}, x_{v}=\hat{x} \cdot \hat{v}, y_{u}=\hat{y} \cdot \hat{u}$, and $y_{v}=\hat{y} \cdot \hat{v}$. Since $\hat{x} \cdot \hat{u}$ is nothing but the cosine of the angle between axes $\hat{x}$ and $\hat{u}$, and similarly for other products, we have the following identities:

$$
\begin{aligned}
& (\hat{x} \cdot \hat{u})^{2}+(\hat{x} \cdot \hat{v})^{2}+(\hat{x} \cdot \hat{w})^{2}=1, \\
& (\hat{y} \cdot \hat{u})^{2}+(\hat{y} \cdot \hat{v})^{2}+(\hat{y} \cdot \hat{w})^{2}=1,
\end{aligned}
$$

and

$$
(\hat{x} \cdot \hat{u})(\hat{y} \cdot \hat{u})+(\hat{x} \cdot \hat{v})(\hat{y} \cdot \hat{v})+(\hat{x} \cdot \hat{w})(\hat{y} \cdot \hat{w})=0 .
$$

Using these relations enables us to write 


$$
\begin{aligned}
& x_{u}^{2}+x_{v}^{2}=1-(\hat{x} \cdot \hat{w})^{2}, \\
& y_{u}^{2}+y_{v}^{2}=1-(\hat{y} \cdot \hat{w})^{2},
\end{aligned}
$$

and

$$
x_{u} y_{u}+x_{u} y_{v}=-(\hat{x} \cdot \hat{w})(\hat{y} \cdot \hat{w}) .
$$

Now $\hat{x} \cdot \hat{w}$ and $\hat{y} \cdot \hat{w}$ are just the $x$ and $y$ components of the surface normal unit vector $\hat{w}$, which for a single-valued height function $h(x, y)$ is simply

$$
\hat{w}=\left(1+h_{x}^{2}+h_{y}^{2}\right)^{-1 / 2}\left(-\hat{x} h_{x}-\hat{y} h_{y}+\hat{z}\right) \text {. }
$$

Therefore,

$$
\begin{aligned}
& \hat{x} \cdot \hat{w}=-h_{x}\left(1+h_{x}^{2}+h_{y}^{2}\right)^{-1 / 2}, \\
& \hat{y} \cdot \hat{w}=-h_{y}\left(1+h_{x}^{2}+h_{y}^{2}\right)^{-1 / 2} .
\end{aligned}
$$

Hence,

$$
\begin{aligned}
m_{u}^{2}+m_{v}^{2}= & \left(1+h_{x}^{2}+h_{y}^{2}\right)^{-1}\left[m_{x}^{2}\left(1+h_{y}^{2}\right)+m_{y}^{2}\left(1+h_{x}^{2}\right)\right. \\
& \left.-2 m_{x} m_{y} h_{x} h_{y}\right] \\
= & {\left[1+(\nabla h)^{2}\right]^{-1}\left[(\nabla m)^{2}+(\nabla m \times \nabla h)^{2}\right] } \\
= & (\nabla m)^{2}-(\nabla m \cdot \nabla h)^{2} /\left[1+(\nabla h)^{2}\right] .
\end{aligned}
$$

${ }^{1}$ M. C. Philips and D. Chapman, Biochim. Biophys. Acta 163, 301 (1986); J. D. Swalen, D. L. Andrade, E. A. Chandross, S. Garoff, J. Israelachvili, T. J. McCarthy, R. Murray, R. F. Pease, J. F. Rabolt, K. J. Wynne, and H. Yu, Langmuir 3, 932 (1987).

${ }^{2}$ C. M. Knobler, Adv. Chem. Phys. 77, 397 (1990); D. Andelman, F. Brochard, C. M. Knobler, and F. Rondelez, in Micelles, Membranes, Microemulsions and Monolayers, edited by W. M. Gelbart, A. BenShaul, and D. Roux (Springer, Berlin, 1993).

${ }^{3}$ A. Halperin, S. Alexander, and I. Schechter, J. Chem. Phys. 86, 6550 (1987); 91, 1383 (1989).

${ }^{4}$ Z. Y. Chen, J. Talbot, W. M. Gelbart, and A. Ben-Shaul, Phys. Rev. Lett. 61, 1376 (1988).

${ }^{5}$ Z.-G. Wang, J. Phys. (Paris) 51, 1431 (1990).

${ }^{6}$ M. E. Costas, Z.-G. Wang, and W. M. Gclbart, J. Chem. Phys. 96, 2228 (1992).

${ }^{7}$ D. Kramer, A. Ben-Shaul, Z.-Y. Chen, and W. M. Gelbart, J. Chem. Phys. 96, 2236 (1992).

${ }^{8}$ A. M. Somoza and R. C. Desai, J. Phys. Chem. 96, 1401 (1992).

${ }^{9}$ W. Helfrich, Z. Naturforsch. Teil C 28, 693 (1973).

${ }^{10}$ S. Marcelja, Biochim. Biophys. Acta 367, 165 (1974).

${ }^{11}$ A. G. Petrov and A. Derzhanski, J. Phys. (Paris) Colloq. 37, C3-155 (1976); A. G. Petrov, M. D. Mitov, and A. Derzhanski, Phys. Lett. A 65, 374 (1978).

${ }^{12}$ J. N. Israelachvili, D. J. Mitchell, and B. W. Ninham, J. Chem. Soc. Faraday Trans. 72, 1525 (1976); D. J. Mitchell and B. W. Ninham, ibid. 77, 601 (1981).
${ }^{13}$ S. A. Safran, L. A. Turkevich, and P. A. Pincus, J. Phys. (Paris) 45, L69 (1984).

${ }^{14}$ Z.-G. Wang and S. A. Safran, J. Phys. (Paris) 51, 185 (1990).

${ }^{15}$ P. Bothorel, J. Belleand, and B. Lemaire, Chem. Phys. Lipids 12, 96 (1974); Biochem. Biophys. Res. Commun. 58, 433 (1974).

${ }^{16}$ E. W. Kaler, A. K. Murthy, B. E. Rodriguez, and J. A. N. Zasadzinski, Science 245, 1371 (1989).

${ }^{17}$ S. A. Safran, P. A. Pincus, and D. Andelman, Science 248, 354 (1990); N. Dan and S. A. Safran, Europhys. Lett. 21, 975 (1993).

${ }^{18}$ F. MacKintosh and S. A. Safran, Phys. Rev. E 47, 1180 (1993).

${ }^{19}$ I. Szleifer, D. Kramer, A. Ben-Shaul, and W. M. Gelbart, Phys. Rev. Lett. 60, 1966 (1988).

${ }^{20}$ F. David and S. Leibler, J. Phys. II France 1, 959 (1991).

${ }^{21}$ S. T. Milner, J.-F. Joanny, and P. Pincus, Europhys. Lett. 9, 495 (1989).

${ }^{22}$ J. H. Schulman and J. B. Montagne, Ann. N.Y. Acad. Sci. 92, 366 (1961).

${ }^{23}$ P.-G. de Gennes and C. Taupin, J. Phys. Chem. 86, 2294 (1982).

${ }^{24}$ S. A. Brazovskii, Zh. Eksp. Teor. Fiz. 68, 175 (1975) [Sov. Phys. JETP 41, 85 (1975)].

${ }^{25}$ T. Garel and S. Doniach, Phys. Rev. B 26, 325 (1982).

${ }^{26}$ D. Andelman, F. Brochard, and J.-F. Joanny, J. Chem. Phys. 86, 3673 (1987).

${ }^{27}$ S. M. Gruner, J. Phys. Chem. 93, 7852 (1989).

${ }^{28}$ P.-G. de Gennes, The Physics of Liquid Crystals (Oxford University, London, 1974).

${ }^{29}$ D. Andelman, T. Kawakasu, and K. Kawasaki, Europhys. Lett. 19, 57 (1992).

${ }^{30}$ A. M. Bibo, C. M. Knobler, and I. R. Peterson, J. Phys. Chem. 95, 5591 (1991).

${ }^{31}$ X. Qiu, J. Ruiz-Garcia, K. J. Stine, C. M. Knobler, and J. V. Selinger, Phys. Rev. Lett. 67, 703 (1991); X. Qiu, J. Ruiz-Garcia, and C. M. Knobler, in Interface Dynamics and Growth, edited by K. S. Liang, M. P. Anderson, R. F. Bruinsma, and D. Scoles (Materials Research Society, Pittsburgh, 1992).

${ }^{32} \mathrm{~J}$. V. Selinger, in Complex Fluids, edited by E. B. Sirota, D. Weitz, T. Witten, and J. Israelachvili (Materials Research Society, Pittsburgh, 1992).

${ }^{33}$ J. V. Selinger, Z.-G. Wang, R. F. Bruinsma, and C. M. Knobler, Phys. Rev. Lett. 70, 1139 (1993).

${ }^{34}$ R. S. Cantor, Macromolecules 14, 1186 (1981); I. Szleifer, D. Kramer, A. Ben-Shaul, W. M. Gelbart, and S. A. Safran, J. Chem. Phys. 92, 6800 (1990); S. T. Milner and T. A. Witten, J. Phys. (Paris) 49, 1951 (1988); L. Leibler, Makromol. Chem., Macromol. Symp. 16, 1 (1988); Z.-G. Wang and S. A. Safran, J. Chem. Phys. 94, 679 (1991); Z.-G. Wang, Macromolecules 25, 3702 (1992).

${ }^{35}$ S. A. Safran, M. O. Robbins, and S. Garoff, Phys. Rev. A 33, 2186 (1986).

${ }^{36}$ V. M. Kaganer, M. A. Osipov, and I. R. Peterson, J. Chem. Phys. 98, 3512 (1993).

${ }^{37}$ See L. D. Landau and E. M. Lifshitz, Statlstical Physics (Pergamon, London, 1980).

${ }^{38}$ J. Toner and D. R. Nelson, Phys. Rev. B 23, 316 (1981); L. Golubovic and Z.-G. Wang, Phys. Rev. Lett. 69, 2535 (1992). 